\title{
Root canal irrigation - which method?
}

\section{Is passive ultrasonic irrigation more effective than syringe irrigation in cleaning the root canal?}

van der Sluis LW, Versluis M, Wu MK, Wesselink PR.

Passive ultrasonic irrigation of the root canal: a review of the literature. Int Endod J 2007; 40:415-26

Data sources Medline and reference lists of relevant articles were consulted.

Study selection Papers were quality assessed by two reviewers: only those addressing passive ultrasonic irrigation (PUI) were included in the review.

Data extraction and synthesis A qualitative synthesis describing the mechanism and effects of PUI is described.

Results Fifty-four papers were included in the review and a qualitative summary of the findings presented.

Conclusions PUI appears to be a possible adjunctive treatment for cleaning the root canal system and may be more effective than syringe irrigation. More research is needed to clarify the underlying physical mechanisms through which PUI works.
Address for correspondence: LWM van der Sluis, Department of Cariology, Endodontology and Pedodontology, Academic Centre for Dentistry Amsterdam ACTA, Louwesweg 1, 1066 EA Amsterdam, The Netherlands. E-mail: 1.vd.sluis@acta.nl

\section{Commentary}

This is an interesting review of the existing literature which attempts to address the practical issues associated with using PUI at the end of root-canal treatment procedure in the hope of obtaining a cleaner root-canal system. It will certainly alert practitioners to the existence of this approach, which may deliver better biologically prepared root canals, although the review does not present enough evidence to disqualify syringe irrigation.

It is well-documented that one of the most important procedures in nonsurgical root-canal treatment and retreatment is chemomechanical preparation of the root canal. ${ }^{1}$ Although intracanal procedures accomplish the mechanical portion, it is vital to adopt a complementary approach for biological preparation. The aim of this is to remove the pulp tissue and/ or micro-organisms there, and also remove any smear layer and dentine debris that occur following and/ or during treatment. Sodium hypochlorite $(\mathrm{NaOCl})$ has been widely accepted as the gold-standard irrigation solution since its introduction in endodontics in 1936. As well as bleaching, deodorising and tissue-dissolving properties, ${ }^{2} \mathrm{NaOCl}$ has been demonstrated to be an effective disinfectant. ${ }^{3}$

The technique of applying $\mathrm{NaOCl}$ to the root canal system does improve the overall effectiveness of biological preparation. Important considerations are, however, the gauge of the needle used, the concentration of the irrigation solution, and the total volume and methods used. ${ }^{4}$ In several studies mentioned in this review, it is not possible to divine all of these details. Also, the in-vitro experimental conditions differ from in-vivo studies in some aspects, among them, the volumes used for disinfection, the accessibility of microbes and the absence of other materials in in-vitro experiments which potentially protect bacteria in vivo. $^{5}$ All of these considerations prevent any conclusion that PUI is more effective than syringe irrigation but it does appears that PUI could be an important adjunct in the management of primary endodontic diseases or root canal failures. There is a need for more experimental evidence and systematic reviews to shed light on this procedure.

\section{Khaled Balto}

Faculty of Dentistry, King Abdulaziz University, Jeddah, Saudi Arabia

1. Haapasalo M, Endal U, Zandi H, Coil JM. Eradication of endodontic infection by instrumentation and irrigation solutions. Endodont Topic 2005; 10:77-102.

2. Gordon TM, Damato D, Christener P. Solvent effects of various dilutions of sodium hypochlorite on vital and necrotic tissue. J Endodont 1981; 7:466-469.

3. Bloomfield SF, Miles GA. The antibacterial properties of sodium dichloroisocyanurate and sodium hypochlorite formulations. J Appl Bacteriol 1979; 46:65-73.

4. Boutsioukis C, Lambrianidis T, Kastrinakis E, Bekiaroglou P. Measurement of pressure and flow rates during irrigation of a root canal ex vivo with three endodontic needles. Int Endodont J 2007; 40:1-10.

5. Tang G, Samaranayake LP, Yip H-K. Molecular evaluation of residual endodontic microorganisms after instrumentation, irrigation and medication. Oral Dis 2004; 6:389-387.

Evidence-Based Dentistry (2007) 8, 5-6. doi:10.1038/sj.ebd.6400509 